\title{
Lipid overload during gestation and lactation can independently alter lipid homeostasis in offspring and promote metabolic impairment after new challenge to high-fat diet
}

Laís Angélica de Paula Simino ${ }^{1}$, Thaís de Fante ${ }^{1}$, Marina Figueiredo Fontana ${ }^{1}$, Fernanda Oliveira Borges ${ }^{1}$, Márcio Alberto Torsoni ${ }^{1}$, Marciane Milanski ${ }^{1}$, Lício Augusto Velloso ${ }^{2}$ and Adriana Souza Torsoni ${ }^{1 *}$

\begin{abstract}
Background: Nutritional status in early life is critically involved in the metabolic phenotype of offspring. However the changes triggered by maternal consumption of high-fat diet (HFD) in pre- or postnatal period should be better understood. Here we evaluated whether maternal HFD consumption during gestation and lactation could differently affect liver miR-122 and miR-370 expression leading to metabolic damages observed in offspring. Moreover, we investigate whether early overnutrition program offspring to more harmful response to HFD in later life.

Methods: Female mice were fed either a standard chow (SC) diet or a HFD three weeks before and during mating, gestation and/or lactation. Offspring were evaluated on the delivery day (d0), in a cross-fostering model at day 28 (d28) and in adult life, after a re-challenge with a HFD (d82).

Results: In vitro analysis using liver cell line showed that palmitate could induced decrease in miR-122 and increase in miR-370 expression. Newborn pups (d0) from obese dams showed a decrease in lipid oxidation markers (Cpt1a and Acadv), an increase in triacylglycerol synthesis markers (Agpat and Gpam), as well as lower miR-122 and higher miR-370 hepatic content that was inversely correlated to maternal serum NEFA and TAG. Pups fostered to SC dams presented an increase in body weight and Agpat/Gpam expression at d28 compared to pups fostered to HFD dams and an inverse correlation was observed between miR-122 hepatic expression and offspring serum TAG. In adult life (d82), the reintroduction of HFD resulted in higher body weight gain and hepatic lipid content. These effects were accompanied by impairment in lipid and glucose metabolism, demonstrated by reduced Cpt1a/Acadvl and increased Agpat/Gpam expression, lower glucose tolerance and insulin sensitivity.
\end{abstract}

Conclusion: Our data suggest that both gestational and lactation overnutrition results in metabolic changes that can permanently alter lipid homeostasis in offspring. The presence of fatty acids in maternal blood and milk seem to be responsible for modulating the expression of miR-122 and miR-370, which are involved in liver metabolism. These alterations significantly increase susceptibility to obesity and ectopic lipid accumulation and lead to a more harmful response to HFD in offspring.

Keywords: microRNAs, Maternal obesity, Metabolic programming, NAFLD

\footnotetext{
* Correspondence: adriana.torsoni@fca.unicamp.br

'Laboratory of Metabolic Disorders, Faculty of Applied Sciences, University of

Campinas -UNICAMP, Limeira, São Paulo, Brazil

Full list of author information is available at the end of the article
} 


\section{Background}

Nutritional status in early or pre-natal life is critically involved in susceptibility to cardiovascular and metabolic diseases development, such as hypertension, dyslipidemia, hyperglycemia and obesity $[1,2]$, events which characterize the metabolic syndrome. Non-alcoholic fatty liver disease (NAFLD) has been considered the hepatic manifestation of this condition and, additionally, is one of the most common causes of liver diseases worldwide [3-5]. The liver plays a central role in modulating glucose homeostasis and lipid metabolism, and NAFLD is caused by an imbalance in lipid metabolism pathways involved in triacylglycerol synthesis, export, delivery, and oxidation $[6,7]$.

Recent studies demonstrated that increased adiposity, hepatic insulin resistance and ectopic fat accumulation have been associated with maternal body weight gain and exposure to a high-fat diet (HFD) along critical phases of development, such as gestation and lactation [8-11]. Moreover, offspring exposed to a HFD in utero and through lactation presents impaired hepatic mitochondrial function and up-regulation of lipogenesis, factors that may contribute to the development of NAFLD and to the progression to a more aggressive liver disease, the non-alcoholic steatohepatitis (NASH) [12].

It is known that lipids can act as signaling molecules and transcriptional activators, and hepatic gene transcription regulation by fatty acids was first reported in 1990s [11, 13, 14]. Saturated fatty acids (SFA), particularly, induce hypothalamic inflammation, endoplasmatic reticulum stress, deleterious effects on blood lipid and lipoprotein profile and, in the liver, can bind to nuclear receptors of transcriptional factors involved in lipid homeostasis and induce lipid droplet accumulation [15, 16].

Perinatal exposure to fatty acids overload, specially SFA, may trigger epigenetic mechanisms that regulate genes involved in lipid sensing and metabolism [11]. MicroRNAs (miRNAs) are epigenetic modulators of gene expression that acts as mRNA silencers, and their regulation are reported to be involved in almost all biological processes in animals $[17,18]$. In contrast, studies have shown that multiple factors can interfere in miRNA expression, such as toxic, chemical and environmental agents and also dietary components [19]. miR-122 and $m i R-370$ participate in the regulation of hepatic lipid metabolism [20-26]. miR-122 is predicted to modulate lipogenic genes and to be potentially targeted by $m i R$ 370 which, in turn, can directly bind to carnitine palmitoyltransferase $1 \alpha(C p t 1 a)$ gene $[20,21]$.

In a recent study, we showed that maternal HFD consumption during pregnancy and lactation leads to a decreased miR-122 and increased miR-370 expression in the liver of recently weaned mice [25]. These miRNAs alterations occurred concurrently with higher expression of lipogenic genes (Gpam, Agpat and Scd1) and lower expression of genes related to fatty acid oxidation (Cpt1a and $A c a d v l$ ) [25]. However, although our previous results reports that $m i R-122$ and $m i R-370$ may participate in the genesis of metabolic damage associated to fatty liver [25], it is not possible to assign the role of gestational or lactational periods to the effects observed in offspring from obese dams and literature data concerning these phenomena are very controversial.

Using a cross-fostering model, Oben and co-workers (2010) showed that lean offspring suckled by obese dams presents increased body weight and food consumption, along with metabolic complications evidenced by increased insulin and leptin levels in plasma and development of NAFLD in adulthood [27]. In contrast, other studies suggest that health status in adulthood is primarily determined by the conditions under which an organism develops in the womb. Gniuli and colleagues [28] showed that exposure to a HFD in utero may lead offspring to a type 2 diabetes phenotype, which could even be transmitted to the progeny. Moreover, maternal consumption of HFD during pregnancy was reported to cause a dysregulation in triglyceride metabolism and, in adipose tissue, to lead to increasing in leptin and suppression of adiponectin levels through epigenetic modifications, leading offspring to a metabolic syndrome-like phenomenon [29].

Importantly, it was previously shown that the metabolic alterations in offspring from HFD fed dams during gestation and suckling period, such as leptin and insulin resistance and ectopic fat accumulation in the liver, persists into adult life, even when they are maintained on a healthy standard chow diet after weaning [10]. However, the molecular mechanism associated to hepatic lipid metabolism modification and the development of fatty liver in adult offspring from HFD fed dams still need to be clarified.

Additionally, another question is still to be answered: which stimulus present at gestational and/or lactational period would be responsible for triggering metabolic programming of offspring from obese dams? The majority of experimental protocols used to study the foetal programming by maternal obesity consist of inducing weight gain through a HFD consumption. During pregnancy, nutrients are transported from maternal circulation to the fetuses via the placenta and, at suckling periods, maternal diet directly affects milk composition [30-34]. Therefore, we hypothesized that the excessive maternal lipids, particularly SFA, could be the trigger for the miRNAs modulation that we earlier reported in the liver of offspring [25].

Thus, the aim of the present study was to test the hypothesis that maternal high fat diet could directly 
modulate $m i R-122$ and $m i R-370$ expression and to investigate the independent contribution of pregnancy and lactation to the altered hepatic lipid metabolism observed in young offspring from obese dams. Additionally, we also evaluated whether this metabolic programming would persist to adulthood and, moreover, whether offspring from HFD-fed dams would present increased metabolic complications after a rechallenge to nutritional overload in adult life.

\section{Methods}

\section{In vitro analysis}

HepG2 human hepatocyte cell line was kindly provided by Dr. Gabriel Forato Anhe, from Farmacology Department of Medical Sciences Faculty of State University of Campinas. Hepa1c1c7 mouse hepatocyte cell line was purchased from BCRJ (Rio de Janeiro Cell Bank, Brazil). HepG2 was maintained in DMEM (Dulbecco's modified Eagle's Medium) and Hepa1c1c7 in $\alpha$-MEM (Minimum Essential Medium Eagle without nucleosides), both supplemented with $10 \%$ FBS, $100 \mathrm{U} / \mathrm{mL}$ penicillin and $0,1 \mathrm{mg} / \mathrm{mL}$ streptomycin, incubated at $37{ }^{\circ} \mathrm{C}$ in $5 \% \mathrm{CO} 2$. Experiments were performed between passages 5 and 40 .

Cells were grown as monolayers at a density of $4 \times$ $10^{5}$ cells $/ \mathrm{mL}$ and two days after seeding, the cultured cells were treated for $6 \mathrm{~h}$ with Palmitate (Sigma Aldrich) $500 \mu \mathrm{M}$ or Palmitate vehicle $(\mathrm{NaOH}+\mathrm{BSA}$, 3:1) as a control.

\section{Animals}

Five weeks old virgin female and male Swiss mice (Mus musculus) were obtained from the Animal Breeding Center at the University of Campinas (Campinas, São Paulo, Brazil) for mating. The general experimental design is illustrated in Fig. 1.

Female mice were randomly fed either HFD or standard chow diet (SC) ad libitum for three weeks before mating (adaptation period). HFD was prepared according to Benatti et al. [25] (Table 1).

Adult males were fed only SC during the adaptation period. Mating was performed by housing females for three days with males. Pregnancy was confirmed by examination of vaginal smears for the presence of sperm.

Pregnant females were maintained in individual polypropylene micro-isolators, at $22 \pm 1{ }^{\circ} \mathrm{C}$ and lights on from 06.00 to $18.00 \mathrm{~h}$. The same diet offered before mating was maintained during gestation period. The study was performed following two protocols: one of them was used to evaluate the effect of maternal overnutrition on offspring metabolism, during gestation or lactation; the other protocol was used to evaluate the metabolic complication in offspring from high fat dams after rechallenge to adult life in nutritional overload (Fig. 1).

To the first protocol on day 0 (d0, first post-partum day), litters were divided in two groups according to maternal diet: offspring from female mice fed SC were called $\mathrm{C}$ and those from female mice fed HFD were called $\mathrm{H}$. Some litters were euthanized and used for $\mathrm{d} 0$ analysis. The remaining litters were adjusted to eight pups each to ensure a standard litter size per mother and were followed until d28 (Fig. 1a).

To study the effect of maternal exposure to HFD on offspring metabolism, during gestation or lactation separately, we carried out the cross-fostering protocol. At d0, individual pups from the litters were randomly assigned to two different foster conditions: (a) unfostered (i.e. the pups were fed by their own mother) and (b) cross-fostered (i.e. the pups were fostered by a mother from the other group). Four groups were obtained: $\mathrm{CC}$ and $\mathrm{HH}$, in which pups were exposed to SC or HFD during gestation and lactation, respectively, and $\mathrm{HC}$ and $\mathrm{CH}$, in which pups were exposed to HFD only during gestation or only during lactation, respectively (Fig. 1b). All pups were weaned on day 18 and separated according to sex. Only males were used to perform the analysis. The cross-fostered ( $\mathrm{CH}$ and $\mathrm{HC})$ and unfostered (CC and $\mathrm{HH}$ ) groups were fed exclusively an $\mathrm{SC}$ diet after weaning until experimental day 28 (d28).

To the second protocol the un-fostered pups from CC and $\mathrm{HH}$ groups were maintained on SC diet after weaning until day 42 (d42). Part of the litters remained on standard chow diet until day $82(\mathrm{CC}$ and $\mathrm{HH})$ and the other part were re-challenged with a HFD until day 82 (CC-HF and HH-HF) (Fig. 1c).

Females in adaptation, gestation or lactational periods were fed growth HFD to ensure an adequate protein supply, while offspring exposed in adult life (CC-HF and HH-HF) were fed maintenance HFD (Table 1).

The total number of animals used in each experiment is mentioned in the figure legends.

\section{Body composition}

Body weight was measured weakly for dams and pups. To estimate the body fat in $\mathrm{d} 0$ groups, we measured naso-anal length of pups, and Lee Index of Obesity (LIO) was calculated using the formula: body weight $(\mathrm{g})$ $(1 / 3)$ / naso-anal length $(\mathrm{cm})$. To measure adiposity in dams, d28, d42, and d82 groups, white adipose tissue (epidydimal and retroperitoneal) was collected and weighted after sacrifice, and the percentage of adiposity normalized by total body weight was calculated.

\section{Food intake}

Food intake was estimated for dams (at gestational day 12 and lactation day 15), d28, d42, and d82 groups 

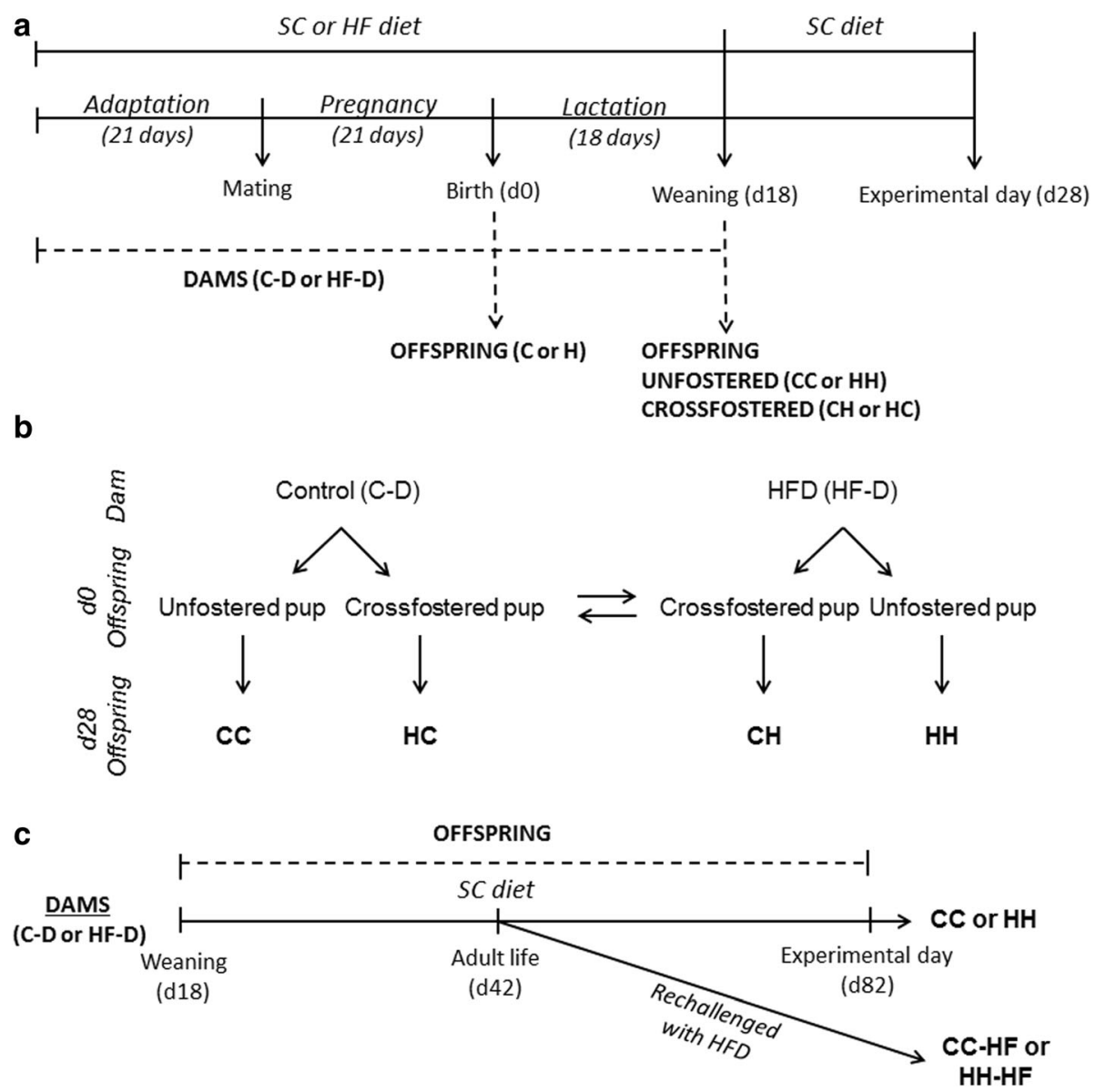

Fig. 1 Experimental Protocol. Experimental protocol followed to investigate the effect of maternal overnutrition on offspring metabolism, during gestation or lactation. Offspring of control (C-D) and HFD (HF-D) dams were evaluated at d0 (C and H) and d28 (CC, CH, HH and HC) (a). Details of the protocol followed to obtain cross-fostered groups (b). Experimental protocol followed to evaluate the metabolic complication in offspring after re-challenge to adult life in nutritional overload at d82 (CC, CC-HF, HH, and $\mathrm{HH}-\mathrm{HF})$ (c)

Table 1 Nutritional composition of the standard chow and experimental diets offered to mice during adaptation, gestation, lactation and post-natal life

\begin{tabular}{llll}
\hline & SC Diet $^{\mathrm{a}}$ & Growth HFD & Maintenance HFD \\
\hline Net Protein (g \%) & $20^{\mathrm{b}}$ & $23,3^{\mathrm{c}}$ & $16,7^{\mathrm{c}}$ \\
Fat Content (g \%) & $4^{\mathrm{d}}$ & $24^{\mathrm{e}}$ & $24^{\mathrm{e}}$ \\
Carbohydrates (g \%) & $65,95^{\mathrm{f}}$ & $42,1^{\mathrm{f}}$ & $49,3^{\mathrm{f}}$ \\
Fiber (g \%) & 5 & 5,55 & 5 \\
Mineral Mix (g \%) & 3,5 & 3,5 & 3,5 \\
Vitamin Mix (g \%) & 1 & 1 & 1 \\
Choline (g \%) & 0,25 & 0,25 & 0,25 \\
Cystine (g \%) & 0,3 & 0,3 & 0,25 \\
Total & 100 & 100 & 100 \\
Energy (KJ/g) & 14,6 & 19,3 & 19,3
\end{tabular}

${ }^{\mathrm{a} N U V I L A B^{\oplus} \mathrm{Cr}-1 \text {; Nuvital }}$

${ }^{b}$ vegetal protein - from wheat and corn (added lysine and methionine)

canimal protein - casein

$\mathrm{d}_{\text {soy oil }}$

esoy oil and lard

starch and saccharose during $24 \mathrm{~h}$ over a period of four days. The average was considered as food intake (Kcal/d).

\section{Biochemical analysis}

To evaluate triglycerides (TAG) and cholesterol (CHOL) levels, blood samples were collected on the experimental days after overnight fasting. By enzymatic colorimetry, serum aliquots were used to measure the levels of TAG and cholesterol.

To evaluate fasting glucose, blood samples were collected after overnight fasting by decapitation or from the tail. Glycaemia was determined on an Accu-Chek Performa glucometer.

To determine serum insulin and leptin, blood samples were collected on the experimental days after overnight fasting. Serum insulin were determined using Rat/Mouse Insulin Kit from Millipore and serum leptin were determined using Mouse Leptin ELISA kit.

Hepatic total lipids content was evaluated as proposed by Folch and colleagues [35]. 
Intraperitoneal Glucose, Insulin and Pyruvate Tolerance Test (GTTip, ITTip and PTTip)

Tolerance tests were performed in adult offspring exposed to a HFD and the same animals were used for all tests, with an interval of at least one week between each test. Glycaemia was determined in an Accu-Chek Performa Glucometer (Roche Diagnostics, Basel, Switzerland). Pyruvate tolerance test was performed to more directly assess the role of HFD in hepatic glucose homeostasis in adult offspring from obese dams.

For the glucose (GTT) and insulin (ITT) tests, mice were starved for $12 \mathrm{~h}$, fed for $\mathrm{h}$ and starved for 4 additional hours before intraperitoneal (IP) injections. For GTT, a solution of $25 \%$ D-glucose was used and each animal was injected with $1 \mathrm{~g} / \mathrm{kg}$ of glucose. Glycaemia was measured at $0,15,30,60,90$ and 120 min after glucose administration. For ITT, recombinant regular insulin $\left(1,5 \mathrm{UI} / \mathrm{kg}\right.$, Humulin ${ }^{\ominus}$ Eli Lilly and Company, EUA) was administrated by IP injection and glycaemia was measured at 0, 3, 6, 9, 12 and 15 min after injection.

For the pyruvate (PTT) test, mice were starved for $12 \mathrm{~h}$ and fed for $2 \mathrm{~h}$, and the test was performed in fed state. IP injection of a pyruvate solution (20\%) was administered in a dose of $2 \mathrm{~g} / \mathrm{kg}$ and glycaemia was measured at $0,15,30,60,90$ and $120 \mathrm{~min}$ after pyruvate administration.

In both GTT and PTT, results are presented as area under curve (AUC) of glycaemia vs. time, above each individual baseline. In ITT, results are presented as kITT, the constant for the glucose clearance rate, calculated using the formula $0,693 / \mathrm{t} 1 / 2$.

\section{Quantitative Real-Time PCR (qRT-PCR)}

Total hepatic RNA was extracted from liver (150 mg), using TRIzol reagent according to the manufacturer's recommendations and quantified using NanoDrop ND2000. Reverse transcription was performed with $3 \mu \mathrm{g}$ of total RNA, using a high-capacity cDNA reverse transcription kit (Thermo Fisher Scientific). Relative expression was determined using a Taqman detection system and primers for the target genes: Agpat1 (Mm 00479699_g1); Acadvl (Mm 00444293_m1); Cpt1a (Mm 01231183_m1); Gpam (Mm 00833328_m1) for liver analysis. Actin beta ( $\beta$-Actin, Mm 02619580_g1) was used as the endogenous control (Thermo Fisher Scientific).

Each PCR contained $20 \mathrm{ng}$ of complementary DNA. Real-time PCR was performed on an ABI Prism 7500 Fast platform. Data were analyzed using the sequence detection system 2.0.5 and expressed as relative values determined by the comparative threshold cycle $(\mathrm{Ct})$ method $(2-\Delta \Delta \mathrm{Ct})$ according to the manufacturer's recommendation.

\section{Purification and quantification of $\mathrm{miR}$}

The miR content was extracted and purified from the Hepa1c1c7 and HepG2 cells and from the liver (150 mg) of the mice using the mirVana miRNA isolation kit (Thermo Fisher Scientific) according to the manufacturer's instructions. The relative expression of $m i R-122$ and miR-370 (ID 002245 and ID 002275, respectively, Thermo Fisher Scientific) was determined using primers with a TaqMan detection system and U6 spliceosomal RNA (ID 001973, Thermo Fisher Scientific) as endogenous controls. Gene expression was accessed by real-time PCR performed on the ABI Prism 7500 Fast platform and analyzed according to 2.5 .

\section{Statistical analysis}

Numeric results are expressed as means with their standard errors of the indicated number of experiments. Student's $t$-test was used for unpaired samples and analysis of variance (ANOVA) for multiple comparisons. A post-hoc test (Bonferroni) was used to determine a significance level of $p \leq 0.05$. Pearson's correlations were used to determine the relation between miR-122 expression and serum TAG or NEFA and a linear regression \pm 95\% confidence interval analysis were performed. In all cases, the statistical significance was set at $p \leq 0.05$. The statistical analyses used in each table and graph are specified in the respective legend.

\section{Results}

In vitro hepatocytes treatment with palmitate alters miR122 and miR-370 levels

To test the hypothesis that fatty acids could modulate the expression of hepatic $m i R-122$ and $m i R-370$, we performed in vitro analysis in mouse (Hepa1c1c7) and human (HepG2) hepatoma cell lines treated with palmitate. The treatment lead to a decreasing in $m i R$ 122 (among 13 to $39 \%$ ) and an increasing in $m i R-370$ levels (among 31 to 114\%; Fig. 2a and b, respectively), indicating that excessive fat could alter the expression of these miRNAs in liver.

\section{Maternal HFD consumption in gestational period leads newborn offspring to altered miRNAs and lipid-related gene expression in the liver}

Next, our goal was to evaluate in which moment - pregnancy or lactation - maternal excessive lipids would be responsible for the modulation of the miRNAs in offspring. For this purpose, we evaluated pups from HF-D and C-D in the day of delivery (d0).

HFD consumption lead dams to alterations in body composition, impaired serum parameters and hepatic total lipids accumulation at gestational period (Additional file 1: Figure S1). Mice from dams fed HFD during gestation $(\mathrm{H})$ showed lower body weight and LIO (Fig. 3a and b, 

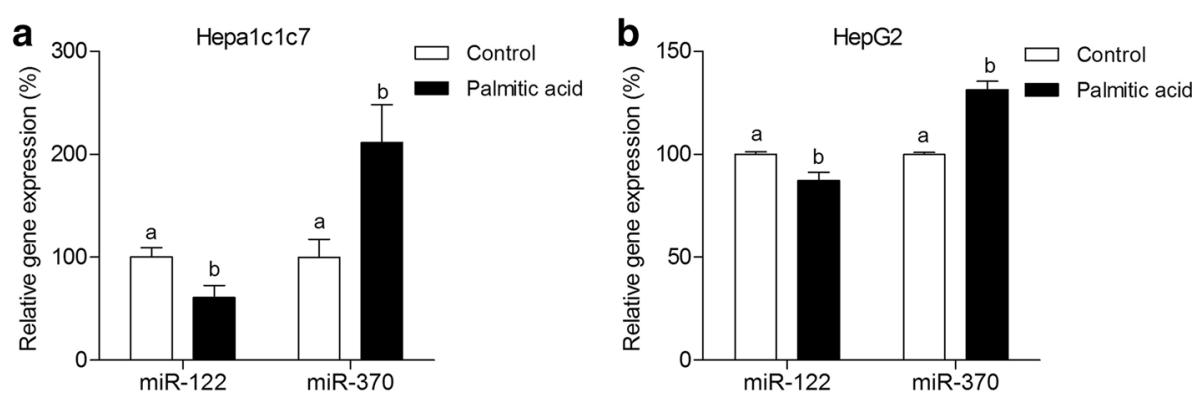

Fig. 2 Fatty acid exposure induces miRNAs modulation in mouse and human liver cell lines. MicroRNA level (qRT-PCR) - miR-122 and miR-370 -

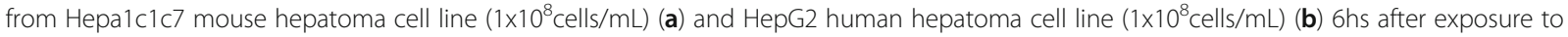
palmitic acid (500uM). For relative gene expression analysis, U6snRNA was used as endogenous control. Values are means ( $n=3-6)+-$ SEM. Student's $t$-test was used to compare Control and Palmitic Acid groups. Different letters indicate statistical significance between groups ( $p \leq 0.05$ )

respectively) in comparison to offspring from control dams (C). The serum levels of CHOL and TAG in $\mathrm{C}$ and $\mathrm{H}$ groups were very similar (Fig. 3c). However, we observed that $\mathrm{H}$ presented higher fasting glucose levels (9\% more than $\mathrm{C}$ ) immediately after birth, but the same insulin levels compared to $\mathrm{C}$ (Fig. 3d and e, respectively).

Mice from $\mathrm{H}$ group had reduced Cpt1a (38.5-fold) and Acadvl (5.6-fold; Fig. 3f) gene expression. An increase in Agpat (1.3-fold) and Gpam (1.9-fold; Fig. 3g) gene expression was also observed in offspring of obese dams.

Moreover, maternal consumption of a HFD during gestation provoked a decrease in $m i R-122(50 \%)$ and an increase in $m i R-370$ (206\%) expression (Fig. 3h). Interestingly, liver $m i R-122$ expression in newborn offspring was inversely correlated with maternal serum TAG levels $(p=0.0016$ - Fig. 3i).

\section{Maternal HFD consumption in gestational or suckling periods independently alters miR-122 and miR-370 and lipid-related gene expression in the liver of recently weaned offspring}

To evaluate whether hepatic modulation of $m i R-122$ and $m i R-370$ by maternal HFD at gestational period would persist after weaning and to investigate if maternal milk would exert a similar effect, we fostered pups after birth.

The alterations observed in body composition and serum biomarkers of HFD-fed dams at gestation persisted to lactation period (Additional file 1: Figure S1). At $\mathrm{d} 28$, we observed an increase in body mass in pups gestated, suckled or both by dams fed HFD compared to those gestated and suckled by control dams, since $\mathrm{CH}$, $\mathrm{HH}$, and $\mathrm{HC}$ showed a higher body weight than $\mathrm{CC}$ (Fig. 4a). However, despite $\mathrm{CH}$ showed a raise in adiposity in comparison to $\mathrm{CC}$, alterations in the gestational period seem to be more harmful to offspring's body composition, since both $\mathrm{HH}$ and $\mathrm{HC}$ groups presented the highest levels of adiposity (Fig. 4b). Caloric intake was elevated in $\mathrm{CH}$ (20\%), $\mathrm{HH}(21 \%)$ and $\mathrm{HC}$ (20\%; Fig. 4c) as well as fasting glucose for the same groups in comparison to $\mathrm{CC}$ (1.6-, 1.7- and 1.7-fold, respectively) (Fig. 4d).

Serum CHOL and TAG levels were higher in $\mathrm{HH}$ in comparison to $\mathrm{CC}$ (1.3-fold). Furthermore, among the cross-fostered mice, $\mathrm{HC}$ showed higher $\mathrm{CHO}$ and TAG levels than $\mathrm{CH}$ (1.3- and 1.5-fold, respectively - Fig. 4e).

The relative expression of oxidative (Cpt1a and $A c a d v l$ ) and lipogenic genes (Agpat and Gpam) in liver was assessed in crossfostered and unfostered offspring. Interestingly Cpt1a expression in $\mathrm{HH}$ was reduced (46\%) compared to CC (Fig. 4f), suggesting reduction in fatty acid oxidation in this group. However, no differences in crossfostered groups were observed. However, Acadvl expression, another marker of fatty acid oxidation, was significantly reduced in unfostered $\mathrm{HH}$ (35\%), as well as in crossfostering groups ( $\mathrm{CH}$ and $\mathrm{HC}$ ) (Fig. 4f).

Additionally, as showed in Fig. 4g, either Agpat or Gpam, both genes involved in triglycerides synthesis pathway, were increased in $\mathrm{HH}(62 \%$ and $33 \%$, respectively) and $\mathrm{CH}$ ( $87 \%$ and $38 \%$, respectively), but they were significantly higher in $\mathrm{HC}$ ( $164 \%$ and $62 \%$, respectively), another indicative of the detrimental effect of diet on gestational period. $\mathrm{HH}$ presented an increasing in hepatic total lipid content (173\%) despite HC have shown a tendency to increasing in this parameter when compared to $\mathrm{CC}$ and $\mathrm{CH}$ (Fig. 4h).

Interestingly, hepatic miR-122 expression was significantly reduced in $\mathrm{CH}$ (2.2-fold), $\mathrm{HH}$ (1.5-fold) and $\mathrm{HC}$ (2.1-fold) compared to $\mathrm{CC}$, while mir-370 expression was significantly increased in $\mathrm{CH}$ (4.4-fold), $\mathrm{HH}$ (7.7fold) and $\mathrm{HC}$ (8.9-fold) (Fig. 4i), corroborating the data showing that HFD feeding at gestational period leads to alterations in these miRNAs in offspring and also showing that excessive lipids consumption at lactation period can also lead to these miRNAs modulation. Moreover, miR-122 expression in the liver of recently weaned offspring showed inversely correlation with serum TAG levels (Fig. 4j). 


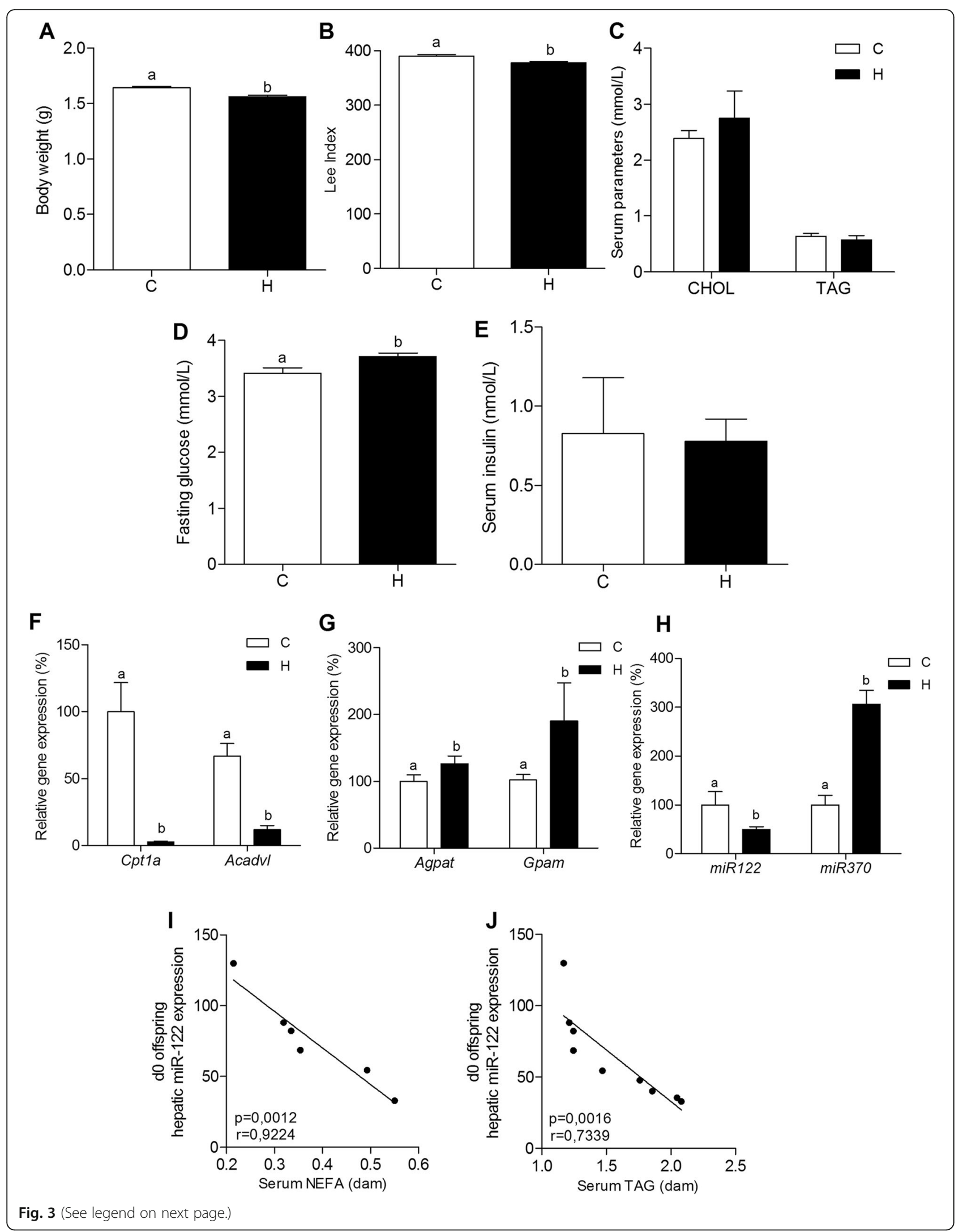


(See figure on previous page.)

Fig. 3 Maternal HFD consumption at gestation period leads newborn offspring to altered miRNAs and lipid metabolism-related gene expression. Body weight (a), lee index of obesity (LIO) (b), serum lipids (CHOL and TAG - c), fasting glucose (d) and serum insulin (e), mRNA levels (qRT-PCR) of hepatic Cptla and Acadvl (f), and Agpat and Gpam (g), microRNA level (qRT-PCR) of hepatic miR-122 and miR-370 (h) from newborn offspring from $\mathrm{C}$ and $\mathrm{H}$ groups. Correlation analysis between hepatic miR-122 from d0 offspring vs. serum NEFA (i) and TAG (j) from dams. For relative gene expression analysis, $\beta$-Actin and U6snRNA were used as endogenous controls. Values are means $(n=5-12)+-$ SEM. Student's $t$-test was used in all analyses to compare $\mathrm{C}$ and $\mathrm{H}$ groups. Different letters indicate statistical significance between groups ( $p \leq 0.05)$

Metabolic programming and hepatic miRNAs modulation by maternal HFD consumption in gestation and lactation persists into adult life of offspring

To investigate whether the metabolic phenotype and hepatic miRNAs modulations observed previously [25] and reported here in $\mathrm{CC}$ and $\mathrm{HH} \mathrm{d} 28$ offspring would persist into adult life, we evaluated the same parameters at $\mathrm{d} 82$. Body composition of $\mathrm{HH}$ remained altered, since body weight $(11.5 \%)$ and adiposity (35\%) were higher in this group in comparison to CC (Fig. 5a and b, respectively), although food intake was not different between the groups (Fig. 5c). Fasting glucose was also higher in $\mathrm{HH}$ (1.4-fold) at d82 (Fig. 5d). Hepatic gene expression analysis showed that, besides Cpt1a did not differ, Acadvl expression was still decreased in $\mathrm{HH}(19 \%$ Fig. 5e) and, moreover, Agpat expression remained highly increased (63\%), although Gpam was not significantly different between $\mathrm{CC}$ and $\mathrm{HH}$ (Fig. 5f). CHOL levels were not changed, but TAG levels were higher (1.1-fold - Fig. 5g) and total hepatic lipids were increased in $\mathrm{HH}$ (1.1-fold - Fig. 5h).

Importantly, the modulation of hepatic miRNAs by maternal HFD consumption at gestation and lactation also persists to adult life, since $\mathrm{HH}$ showed lower $m i R$ 122 (42\%) and higher $m i R-370$ (139\%) expression in comparison to $\mathrm{CC}$ at $\mathrm{d} 82$ (Fig. $5 \mathrm{i}$ ).

\section{Maternal HFD consumption at gestation and lactation leads offspring to increased metabolic complications when exposed to a HFD in adult life}

Finally, we evaluated whether an HFD exposure in early life and its consequent modulation of key hepatic miRNAs would increase HFD sensitivity in later life and negatively affect lipid and glucose metabolism in offspring, we used a model in which offspring from obese and lean dams were re-challenged to HFD.

At d42, we investigated some parameters of offspring prior to exposure to HFD in order to observe whether the phenotype of $\mathrm{HH}$ group remained different from $\mathrm{CC}$ group, as observed on $\mathrm{d} 28$. Compared to $\mathrm{CC}, \mathrm{HH}$ were still heavier (10\%) and presented higher fat mass (38\%) (Additional file 2: Figure S2A and B, respectively), although caloric intake between groups was not different (Additional file 2: Figure S2C). HH presented impaired glucose homeostasis with more elevated fasting glucose (1.3-fold) and insulin (1.2-fold) than CC (Additional file 2:
Figure S2D and E, respectively). Moreover, TAG levels were higher in $\mathrm{HH}$, despite $\mathrm{CHOL}$ serum levels were not different between the groups (Additional file 2: Figure S2F).

After HFD exposure for over five weeks, HH-HF presented a significantly higher weight gain, as early as the first week of HFD consumption, and an increased total body mass (11\%) in comparison to CC-HF (Fig. 6a and $\mathrm{b}$, respectively). Adiposity was also increased in $\mathrm{HH}-\mathrm{HF}$ (35\%), as well as food intake (1.2-fold) (Fig. 6c and d, respectively).

Serum parameters reveals higher levels of $\mathrm{CHOL}$, TAG, leptin and fasting glucose in $\mathrm{HH}-\mathrm{HF}$ (Fig. 6e and f) and, moreover, tolerance tests showed that $\mathrm{HH}-\mathrm{HF}$ presented disturbances in glucose homeostasis, since these group presented higher AUC in GTT (45\%) and PTT (36\%) and diminished glucose clearance as shown in kITT (5.7-fold - Fig. 6g, h and i, respectively).

Besides no differences were observed in Acadvl, Cpt1a expression was decreased in HH-HF liver (Fig. 6j) and, furthermore, Agpat and Gpam expression were markedly increased (242\% and 161\%, respectively - Fig. 6k). Additionally, HH-HF presented higher levels of hepatic total lipid content when in consumption of a HFD than CC-HF (18.5\% - Fig. 6l).

\section{Discussion}

Maternal consumption of HFD during critical developmental periods has been shown to lead to commitments on offspring's homeostasis and may result in metabolic disorders [11, 28]. We recently showed that maternal consumption of HFD during gestation and lactation activates pro-inflammatory pathways and unfolded protein response, impairs cholinergic anti-inflammatory pathway, modulates autophagy proteins and also affects lipid metabolism in offspring [25, 36-38]. Offspring from HFD-fed dams present downregulation of hepatic $\beta$-oxidation-related genes and upregulation of genes involved in lipid synthesis, and these alterations seem to be driven by the modulation of miR-122 and miR-370 levels, causing metabolic adaptations that lead to increased ectopic lipid accumulation in the liver of recently weaned mice [25].

Despite some studies have suggested that maternal macronutrients may be responsible for gene expression modulation in foetal offspring [39-41], we have not 


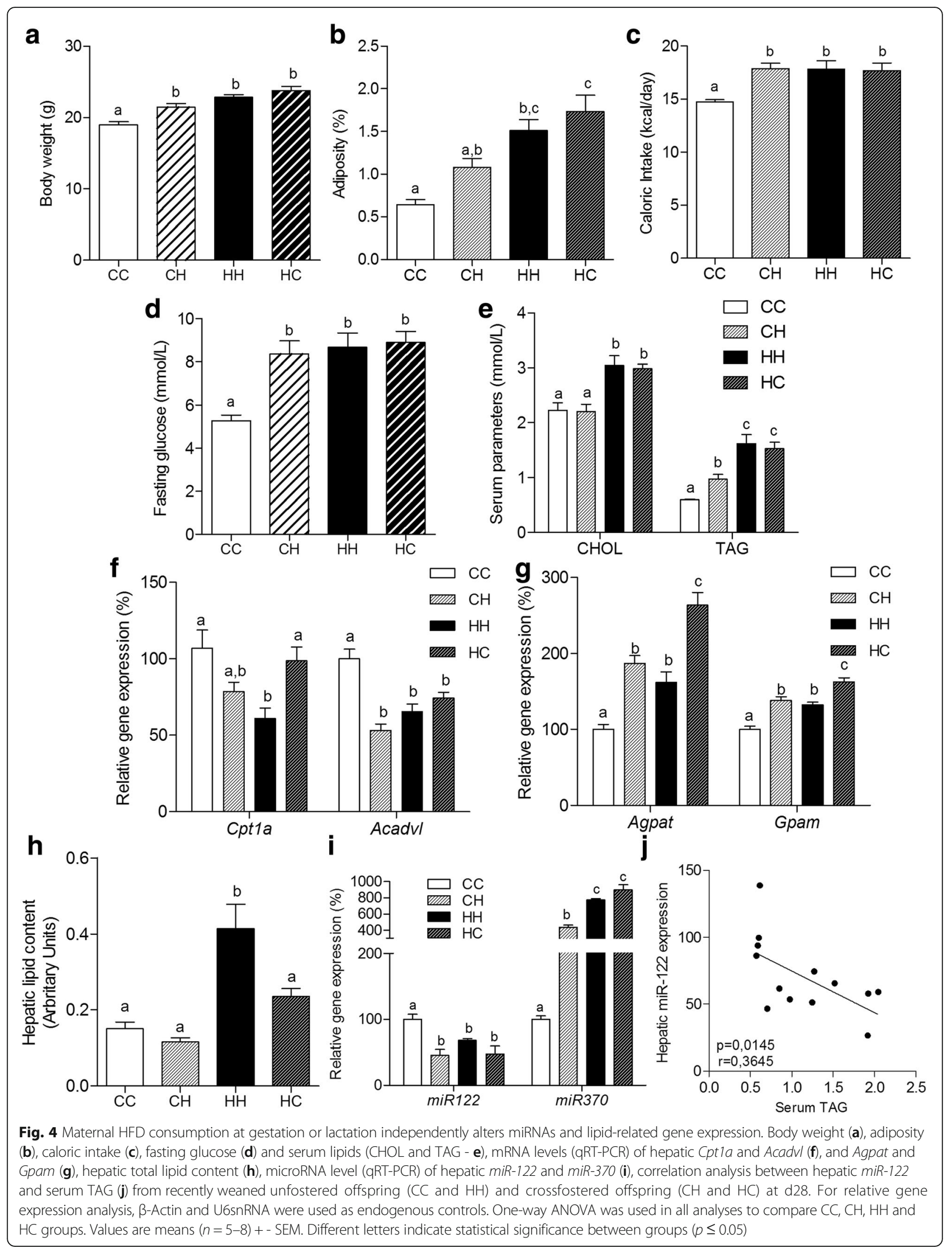




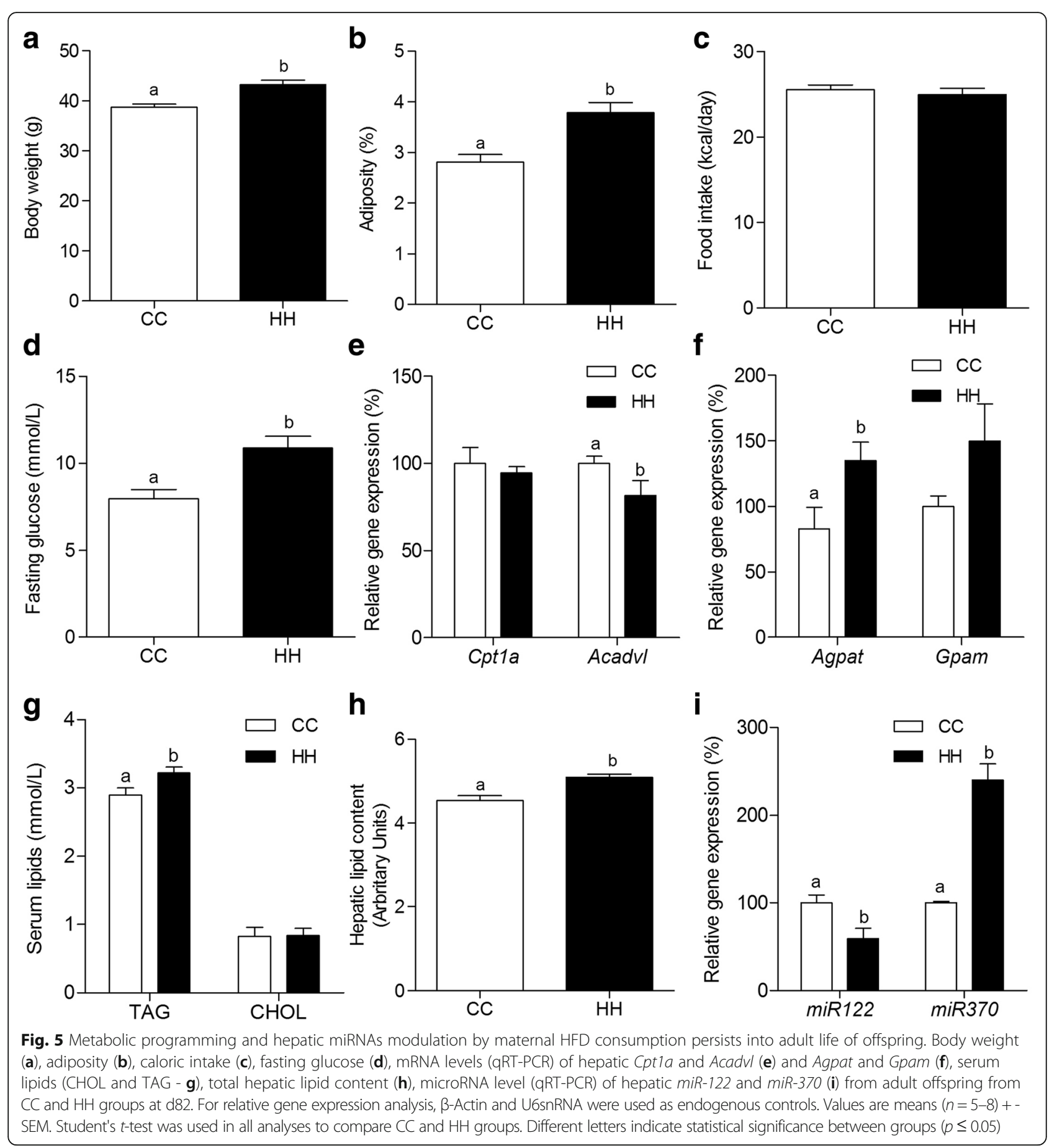

found studies showing that components of maternal diet could directly alter miRNAs expression in offspring. It is known that a HFD consumption increases lipids levels in serum, such as TAG, CHOL and free fatty acids (FFA) [25] and, moreover, maternal diet can directly affect breast milk composition [32-34, 42]. Therefore we hypothesized that the lipids consumed by the dams, specially SFA, could be responsible for $m i R-122$ and miR-370 modulation in the liver of offspring through placental delivery during gestation and/or milk composition during suckling period.

As shown in the present study, treatment with palmitic acid, one of the most abundant SFAs in the human diet and blood, leads to a decrease in miR-122 and increased $m i R-370$ levels in hepatocytes. It have been largely reported that SFAs play an important role in the development of insulin resistance by their directly regulation of inflammatory and metabolic pathways [15, 16, 43]. 


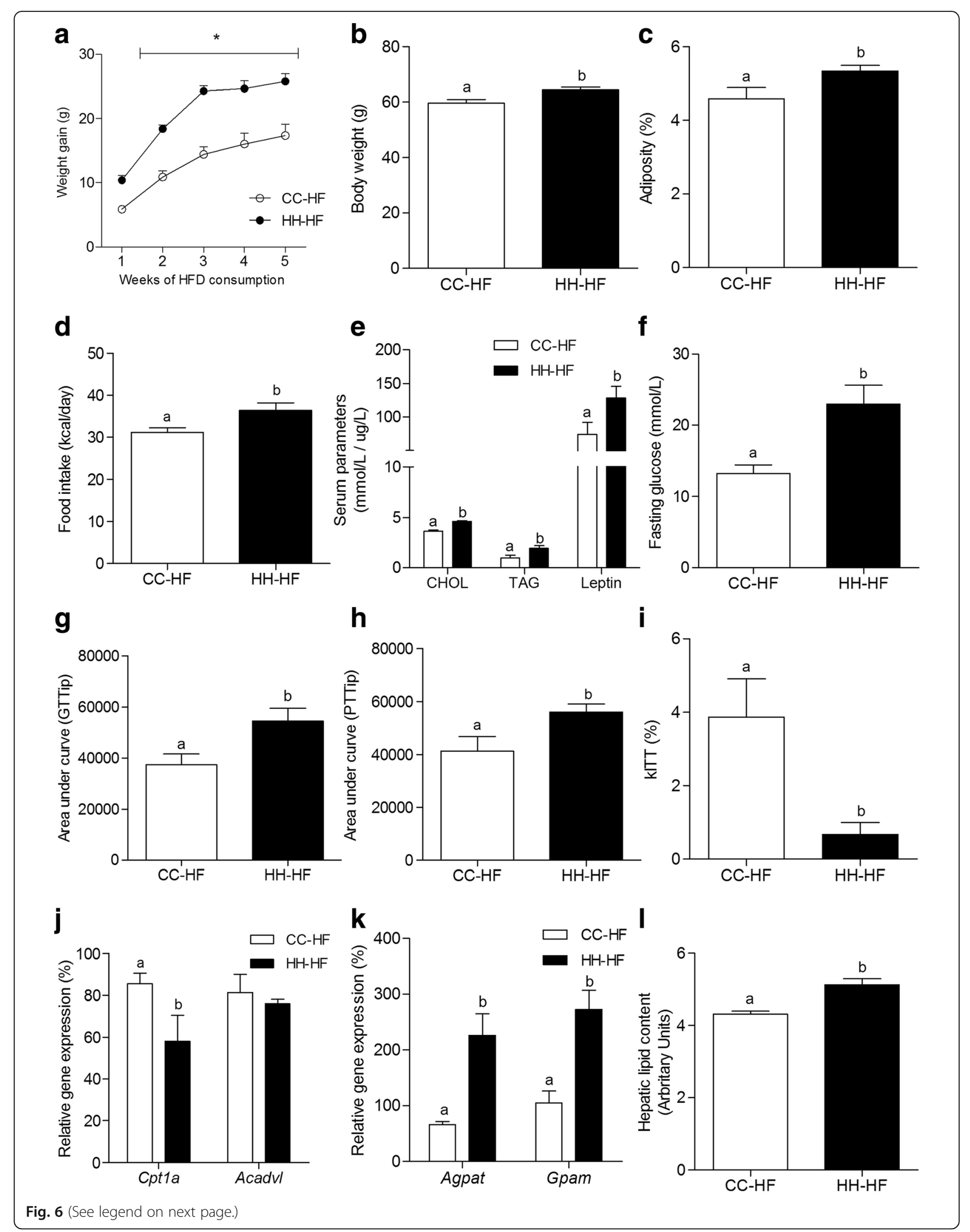


(See figure on previous page.)

Fig. 6 Maternal HFD consumption leads offspring to increased metabolic complications when exposed to HFD in adult life. Weight gain (a), body weight (b), adiposity (c), caloric intake (d), serum parameters (CHOL, TAG and leptin) (e), fasting glucose (f), area under curve (AUC) of GTT (g) and PTT (h), glucose clearance during ITT (i), mRNA levels (qRT-PCR) of hepatic Cpt1a and Acadvl (j) and Agpat and Gpam (k), total hepatic lipid content (I) from adult offspring after exposure to HFD for 40 days at d82 (CC-HF and HH-HF groups). For relative gene expression analysis, $\beta$-Actin was used as endogenous control. Values are means $(n=5-8)+-$ SEM. Student's $t$-test was used in all analyses to compare CC-HF and HH-HF groups. Different letters indicate statistical significance between groups ( $p \leq 0.05)$

More recently, studies showed that several miRNAs are dysregulated in rodent models of diet-induced obesity, type 2 diabetes and NAFLD [44], reinforcing that excessive fat consumption, particularly SFAs, may be involved in miRNAs modulation that participate in the genesis of metabolic diseases. Importantly, in vitro analysis showed that miRNAs can be direct targets of dietary components, including fatty acids $[19,45]$. Moreover, Nie and colleagues (2014) induced lipid accumulation in human hepatocyte cell line using oleic acid. In this steatotic hepatocyte model, miR-122 expression was downregulated and, importantly, transfection with miR-122 mimic significantly reduced lipids within the hepatocytes [46].

After confirming that the in vitro treatment with palmitate was able to reduce $m i R-122$ and increase $m i R$ 370 expression, we aimed to investigate the independent contribution of maternal excessive lipids consumption at gestational or lactation periods on the modulation of these miRNAs, since only few studies have investigated the relative contribution of pre- or post-natal maternal HFD consumption to metabolic phenotype in offspring. Sun and colleagues [47] showed that maternal HFD consumption during the suckling period has a high influence on leptin signaling in offspring. On the other hand, Cerf and colleagues [48] showed that neonates exposed to HFD only during foetal development presented reduced volume and number of $\beta$-cells, accompanied by sustained and irreversible hyperglycaemia during adulthood.

Here, we showed that pups from HFD-fed dams presents lower body weight and LIO at birth (d0), in comparison to pups from control dams. Similar studies have shown higher body weight and macrosomia in newborns from obese dams $[49,50]$ however, some authors have shown reduced body weight in offspring at d0 using models of maternal hyperglycemia and transient hyperinsulinemia. In our model, HFD-fed dams presented higher insulin levels prior to mating when compared to control dams and, although this hormone does not cross the placental barrier, some authors suggest that maternal hyperinsulinemia may lead to placental disorders and that this occurrence may be related to the delayed foetus development [51-53].

Interestingly, besides the lower body weight, hepatic lipid synthesis seems to be increased in offspring from HFD-fed dams at birth, as suggested by liver Agpat and
Gpam levels and, in contrast, genes related to fatty acid oxidation (Cpt1a and Acadvl) are reduced in their liver. There are several evidences in humans indicating that maternal obesity leads to increased fuel availability for the foetus and may drive to increased hepatic fat storage [54]. Maternal nutritional overload and placental transfer is a challenge to foetal development and, as suggested by Brumbaugh and Friedman [54], until final gestational phases, subcutaneous fat is not available to act as a storage buffer so, under dietary excesses, the foetal liver, among another organs, becomes a fat deposit. McCurdy and co-workers [55] showed, in non-human primates, an increase in hepatic TAG and elevated hepatic expression of gluconeogenesis key genes in foetus from HFD-fed dams at gestational third trimester and the maternal resistance to obesity development did not reduce the effects of the HFD-consumption on hepatic metabolism of the fetuses. Therefore, these findings suggest that maternal HFD consumption during pregnancy can modulate lipid metabolism in offspring, favoring fat deposition.

As we have speculated, miR-122 and miR-370 may be involved in the impairments of lipid homeostasis of newborns from HFD-fed dams, since we find here that these miRNAs are modulated at $\mathrm{d} 0$, as early as the hepatic enzymes involved in lipid metabolism. Furthemore, maternal FFA at gestation correlated directly with $m i R-370$ and inversely with miR-122 in the liver of newborns, thus reinforcing the hypothesis that maternal lipids that cross placental barrier are able to modulate miRNAs expression of offspring.

It have been shown that mice lacking the gene encoding $m i R-122 a$ are viable, but they develop hepatosteatosis, NASH and fibrosis and present hepatic infiltration of inflammatory cells that produce pro-tumorigenic cytokine, including IL- 6 and TNF $\alpha$, thus leading to the development of hepatocellular carcinoma $[22,26,56]$. Besides, Hsu and colleagues (2012) showed that knockout mice for miR-122 in liver present higher expression of several hepatic enzymes involved in TAG synthesis, including Agpat [56]. Additionally, Xu and colleagues showed that inhibition of miR-370 led to downregulation of pro-inflammatory cytokines, suggesting that this miRNA plays a pro-inflammatory role and may be related to hepatic damage [57]. Although the relationship between the levels of miR-122 and miR-370 is still subject to debate $[14,19]$, we showed that there is an 
inverse expression of these miRNA in our model at different ages. Thus, considering the impact of lipid metabolism and inflammatory signaling on the development of NAFLD, maternal HFD consumption during the gestational period could be considered an important risk factor for liver diseases in offspring.

Using the cross-fostering model, we were able to further confirm the importance of maternal HFD consumption during gestation to hepatic lipid metabolism and also investigate whether the suckling period would exert the same effect. Surprisingly, miR-122 and miR-370 levels seem to be also modulated by maternal milk, since pups from control dams suckled by HFD-fed dams showed a decrease in miR-122 and increased expression of hepatic miR-370 as well as upregulation of TAG synthesis markers (Agpat and Gpam) and downregulation of a fatty oxidation marker (Acadvl). Regardless of the negative effects of HFD consumption at gestational period seem to be more pronounced, in general, the impairments in lipid homeostasis observed at gestation are also present at the suckling period by itself. Although we did not evaluate milk composition, previous studies demonstrated that HFD consumption and mainly the availability of plasma fatty acid for uptake by the mammary gland alters the fatty acid composition and content, specially medium chain fatty acids secreted in milk of human and rat $[42,58,59]$, and may exert effects on the fat accumulation in the neonatal liver.

Besides, offspring from HFD-dam presents altered serum lipid levels [25] and here, offspring's serum TAG correlated directly with $m i R-370$ and inversely with miR122 hepatic expression.

Given that we showed that excess maternal lipids at both gestational and lactation periods are independently able to alter hepatic miRNAs in newborn and recently weaned offspring, respectively, that may lead to impaired lipid metabolism, the next step was to investigate whether these modulation would persist into adult life. As shown here and previously, offspring exposed to maternal HFD at gestation and lactation still present, at $\mathrm{d} 82$, increased body weight, adiposity, fasting glucose and fat accumulation within the liver. Interestingly, adult offspring from HFD-fed dam still shows decreased $m i R$ 122 and increased miR-370 expression in the liver. These findings suggest that epigenetic modifications at pre and recent post-natal life are persistent. Thorn and colleagues (2014) also showed that, in non-human primates, maternal insulin resistance induced by HFD consumption at developmental stages negatively and irreversible affects hepatic immune system and development of de novo lipogenic pathways [60]. For these reasons, authors believes that exposure to excess maternal lipids at critical development periods may be considered the "first hit" for the pathogenesis of liver diseases [60, 61].
The "two hits" or "multiple hits" hypothesis was postulated as an attempt to explain the mechanisms underlying the progression of NAFLD to more aggressive liver diseases, such as NASH and fibrosis. Ectopic accumulation of fat in the liver, the steatosis, was considered the "first hit", and authors suggested that a second hepatic insult, such as oxidative stress or drugs, would be necessary to lead to chronical inflammation and NAFLD progression [62, 63]. Nowadays, it is hypothesized that the obesity and lipid overload in intra-uterine environment could program the foetal liver and trigger the "first hit" and, afterwards, a high-fat diet consumption in postnatal life would be enough to lead to hepatic inflammation and to liver diseases progression [61].

In this context, we showed here that offspring challenged with maternal lipid overload in pre-natal life and through lactation present a more deleterious response when exposed to a HFD in adult life, by showing an increase in weight gain, adiposity and serum parameters, such as CHOL, TAG, leptin and fasting glucose and, moreover, exacerbated glucose homeostasis disturbances and lipid accumulation in the liver, factors that may lead to inflammatory pathways activation. We showed recently that glucose homeostasis disturbances observed in adult offspring re-challenged to HFD may occur due to an imbalance in insulin signaling in peripheral tissues (such as visceral adipose tissue) and hypothalamus. Additionally a failure in glucose production blockade in the liver was also observed [64]. Therefore, since insulin resistance plays a central role in the metabolic syndrome and the ectopic lipid accumulation in the liver has been considered the hepatic manifestation of this condition $[4,5]$, our results suggest that offspring from obese dams that are re-exposed to a HFD in adult life could present a metabolic syndrome-like phenotype.

Besides others studies also had shown these increased metabolic disturbances in adult offspring from obese dams exposed to a HFD in adult [65], to our knowledge, the present study was the first to show that maternal diet leads to persistent modulation of key liver miRNAs that may be involved in the development of insulin resistance and NAFLD. Thus, the attention to maternal diet during gestation and lactation seems to be of great importance to avoid permanent changes during foetal development that could contribute to the development of obesity and its comorbidities in later life.

\section{Conclusions}

In summary, maternal HFD consumption during gestation and lactation affect the metabolic phenotype in the offspring. The presence of fatty acids in maternal blood and milk seem to be responsible for modulating the expression of liver miR-122 and $m i R-370$, which are involved in liver homeostasis. These findings provide 
important information regarding the effects of maternal nutrition, especially during gestation, on the metabolic phenotype of offspring. However, further studies are necessary to understand how nutritional intervention during gestational and lactation periods can contribute to reduce metabolic damage in offspring.

\section{Additional files}

Additional file 1: Figure S1. Antropometric and biochemical parameters of dams at gestation and lactation. Body weight (A), adiposity (B), fasting glucose $(C)$, serum insulin (D) and lipids (CHOL and TAG - E) and total hepatic lipid content (F) of control (C-D) and HFD (HF-D) dams in the gestational day 12 , and lactational day 15 . Values are means $(n=3-6)+-$ SEM. Student's $t$-test was used in all analyses to compare C-D and HF-D groups in each period (gestation or lactation). Different letters indicate statistical significance between groups ( $p \leq 0.05)$. (JPG $667 \mathrm{~kb}$ )

Additional file 2: Figure S2. Characterization of $d 42$ offspring from control and obese dams prior to HFD exposure. Body weight (A), adiposity (B), caloric intake (C), fasting glucose (D), serum insulin (E) and lipids (CHOL and TAG - F), in d42 CC and HH groups. Values are means $(n=4-8)+-$ SEM. Student's $t$-test was used in all analyses to compare CC and $\mathrm{HH}$ groups. Different letters indicate statistical significance between groups $(p \leq 0.05)$. (JPG $390 \mathrm{~kb})$

\section{Abbreviations}

Acadvl: Acyl-CoA dehydrogenase very long-chain; Agpat: 1-acylglycerol-3phosphate acyltransferase; CHOL: Cholesterol; Cpt1a: Carnitine palmitoyltransferase 1a; FFA: Free fatty acid; Gpam: Glycerol-3-Phosphate acyltransferase mitochondrial; HFD: High-fat diet; IL-6: Interleukin 6; miR/miRNA: microRNA; NAFLD: Non alcoholic fatty liver disease; NASH: Non alcoholic steatohepatitis; NEFA: Non-esterified fatty acid; Scd1: Stearoyl-CoA desaturase 1; SFA: Saturated fatty acid; TAG: Triacylglycerol; TNFa: Tumor necrosis factor a

\section{Funding}

The present study was supported by grant \#2011/22156-7, \#2013/07607-8 and \#2014/18165-9, São Paulo Research Foundation (FAPESP). Laboratories of Cell Signaling and Metabolic Disorders belongs to the Obesity and Comorbidities Research Center. The authors state that the funding agency was responsible only for financial support, and the experimental design, collection, analysis and interpretation of data was independent of support from FAPESP.

\section{Availability of data and materials}

The datasets obtained and/or analyzed during the current study are available from the corresponding author on reasonable request.

\section{Authors' contributions}

LAPS was involved in the development of the study hypothesis, reviewed the literature, conducted data collection, managed the statistical analysis, provided results interpretation, drafted and edited the manuscript. TF, MFF and $\mathrm{FOB}$ contributed to the development of the study hypothesis and to data collection, and provided advice regarding interpretation of the results. MAT, MM and LAV provided advice regarding interpretation of the results and assisted with the manuscript edition. AST was responsible for the study design, provided interpretation of the results, drafted and edited the manuscript. All authors read and approved the final manuscript.

\section{Competing interests}

The authors declare that they have no competing interests.

\section{Ethics approval}

All experiments were performed in accordance with the recommendations of the Brazilian College for Animal Experimentation (COBEA) guidelines and approved by the Ethical Committee for Animal Use, Protocol no. 3175-1, State University of Campinas - UNICAMP (Campinas, São Paulo, Brazil).

\section{Author details}

Laboratory of Metabolic Disorders, Faculty of Applied Sciences, University of Campinas -UNICAMP, Limeira, São Paulo, Brazil. ' Laboratory of Cell Signaling, Faculty of Medical Sciences, University Of Campinas - UNICAMP, Campinas, São Paulo, Brazil.

Received: 27 October 2016 Accepted: 6 February 2017

Published online: 20 February 2017

\section{References}

1. Waterland RA, Garza C. Potential mechanisms of metabolic imprinting that lead to chronic disease. Am J Clin Nutr. 1999;69:179-97.

2. Vogt MC, Paeger L, Hess S, et al. Neonatal Insulin Action Impairs Hypothalamic Neurocircuit Formation in Response to Maternal High-Fat Feeding. Cell. 2014; 156:495-509. doi:10.1016/j.cell.2014.01.008.

3. Angulo P. Obesity and nonalcoholic fatty liver disease. Nutr Rev. 2007;65: S57-63.

4. Bugianesi E, Moscatiello S, Ciaravella MF, Marchesini G. Insulin resistance in nonalcoholic fatty liver disease. Curr Pharm Des. 2010;16:1941-51.

5. Bugianesi E, McCullough AJ, Marchesini G. Insulin resistance: A metabolic pathway to chronic liver disease. Hepatology. 2005;42:987-1000. doi:10. 1002/hep.20920.

6. Samuel VT, Liu Z-X, Qu X, et al. Mechanism of Hepatic Insulin Resistance in Non-alcoholic Fatty Liver Disease. J Biol Chem. 2004;279:32345-53. doi:10. 1074/jbc.M313478200.

7. Yamaguchi K, Yang L, McCall S, et al. Inhibiting triglyceride synthesis improves hepatic steatosis but exacerbates liver damage and fibrosis in obese mice with nonalcoholic steatohepatitis. Hepatology. 2007;45:1366-74. doi:10.1002/hep.21655.

8. Samuelsson A-M, Matthews PA, Argenton M, et al. Diet-induced obesity in female mice leads to offspring hyperphagia, adiposity, hypertension, and insulin resistance: a novel murine model of developmental programming. Hypertension. 2008;51:383-92. doi:10.1161/HYPERTENSIONAHA.107.101477.

9. Elahi MM, Cagampang FR, Mukhtar D, et al. Long-term maternal high-fat feeding from weaning through pregnancy and lactation predisposes offspring to hypertension, raised plasma lipids and fatty liver in mice. $\mathrm{Br} J$ Nutr. 2009:102:514. doi:10.1017/S000711450820749X.

10. Ashino NG, Saito KN, Souza FD, et al. Maternal high-fat feeding through pregnancy and lactation predisposes mouse offspring to molecular insulin resistance and fatty liver. J Nutr Biochem. 2012;23:341-8. doi:10.1016/j. jnutbio.2010.12.011.

11. Heerwagen MJR, Miller MR, Barbour LA, Friedman JE. Maternal obesity and fetal metabolic programming: a fertile epigenetic soil. AJP Regul Integr Comp Physiol. 2010;299:R711-22. doi:10.1152/ajpregu.00310.2010.

12. Bruce KD, Cagampang FR, Argenton $M$, et al. Maternal high-fat feeding primes steatohepatitis in adult mice offspring, involving mitochondrial dysfunction and altered lipogenesis gene expression. Hepatology. 2009;50: 1796-808. doi:10.1002/hep.23205.

13. Blake WL, Clarke SD. Suppression of rat hepatic fatty acid synthase and S14 gene transcription by dietary polyunsaturated fat. J Nutr. 1990;120:1727-9.

14. Jump DB, Tripathy S, CMD. Fatty Acid-Regulated Transcription Factors in the Liver. Annu Rev Nutr. 2013;33:249-69. doi:10.1146/annurev-nutr-071812-161139.

15. Milanski M, Degasperi G, Coope A, et al. Saturated fatty acids produce an inflammatory response predominantly through the activation of TLR4 signaling in hypothalamus: implications for the pathogenesis of obesity. J Neurosci. 2009;29:359-70. doi:10.1523/JNEUROSCI.2760-08.2009.

16. Masi LN, Rodrigues AC, Curi R. Fatty acids regulation of inflammatory and metabolic genes. Curr Opin Clin Nutr Metab Care. 2013;16:418-24. doi:10. 1097/MCO.0b013e32836236df

17. Rottiers V, Näär AM. MicroRNAs in metabolism and metabolic disorders. Nat Rev Mol Cell Biol. 2012;13:239-50. doi:10.1038/nrm3313.

18. Ha M, Kim VN. Regulation of microRNA biogenesis. Nat Rev Mol Cell Biol. 2014;15:509-24. doi:10.1038/nrm3838.

19. Izzotti A, Cartiglia C, Steele VE, De Flora S. MicroRNAs as targets for dietary and pharmacological inhibitors of mutagenesis and carcinogenesis. Mutat Res - Rev Mutat Res. 2012;751:287-303. doi:10.1016/j.mrrev.2012.05.004.

20. Niopoulos D, Drosatos K, Hiyama Y, et al. MicroRNA-370 controls the expression of microRNA-122 and Cpt1alpha and affects lipid metabolism. J Lipid Res. 2010;51:1513-23. doi:10.1194/jlr.M004812.

21. Li ZY, Xi Y, Zhu WN, et al. Positive regulation of hepatic miR-122 expression by HNF4a. J Hepatol. 2011;55:602-11. doi:10.1016/j.jhep.2010.12.023. 
22. Tsai WC, Da Hsu S, Hsu CS, et al. MicroRNA-122 plays a critical role in liver homeostasis and hepatocarcinogenesis. J Clin Invest. 2012;122:2884-97. doi:10.1172/JCl63455.

23. Wen J, Friedman JR. miR-122 regulates hepatic lipid metabolism and tumor suppression. J Clin Invest. 2012;122:2773-6. doi:10.1172/JCl63966.

24. Yang YM, Seo SY, Kim TH, Kim SG. Decrease of microRNA-122 causes hepatic insulin resistance by inducing protein tyrosine phosphatase $1 \mathrm{~B}$, which is reversed by licorice flavonoid. Hepatology. 2012;56:2209-20. doi:10.1002/hep.25912.

25. Benatti RO, Melo AM, Borges FO, et al. Maternal high-fat diet consumption modulates hepatic lipid metabolism and microRNA-122 (miR-122) and microRNA-370 (miR-370) expression in offspring. Br J Nutr. 2014;111:211222. doi:10.1017/S0007114514000579.

26. Nakao K, Miyaaki H, Ichikawa T. Antitumor function of microRNA-122 against hepatocellular carcinoma. J Gastroenterol. 2014;49:589-93. doi:10.1007/ s00535-014-0932-4.

27. Oben JA, Mouralidarane A, Samuelsson A-M, et al. Maternal obesity during pregnancy and lactation programs the development of offspring nonalcoholic fatty liver disease in mice. J Hepatol. 2010;52:913-20. doi:10.1016/j. jhep.2009.12.042

28. Gniuli D, Calcagno A, Caristo ME, et al. Effects of high-fat diet exposure during fetal life on type 2 diabetes development in the progeny. J Lipid Res. 2008;49:1936-45. doi:10.1194/jlr.M800033-JLR200.

29. Masuyama $\mathrm{H}$, Hiramatsu Y. Effects of a high-fat diet exposure in utero on the metabolic syndrome-like phenomenon in mouse offspring through epigenetic changes in adipocytokine gene expression. Endocrinology. 2012; 153:2823-30. doi:10.1210/en.2011-2161.

30. Tarrade A, Panchenko P, Junien C, Gabory A. Placental contribution to nutritional programming of health and diseases: epigenetics and sexual dimorphism. J Exp Biol. 2015;218:50-8. doi:10.1242/jeb.110320.

31. Brett K, Ferraro Z, Yockell-Lelievre J, et al. Maternal-Fetal Nutrient Transport in Pregnancy Pathologies: The Role of the Placenta. Int J Mol Sci. 2014;15: 16153-85. doi:10.3390/ijms150916153.

32. Prado M Del, Delgado G, Villalpando S. Maternal Lipid Intake During Pregnancy and Lactation Alters Milk Composition and Production and Litter Growth in Rats. J Nutr. 1997;3:458-462.

33. Oosting A, Verkade HJ, Kegler D, et al. Rapid and selective manipulation of milk fatty acid composition in mice through the maternal diet during lactation. J Nutr Sci. 2015;4:e19. doi:10.1017/jns.2015.13.

34. Bautista CJ, Montaño S, Ramirez V, et al (2016) Changes in milk composition in obese rats consuming a high-fat diet. Br J Nutr 538-546. doi: 10.1017/ S0007114515004547

35. Folch J, Lees M, Sloane Stanley GH. A simple method for the isolation and purification of total lipides from animal tissues. J Biol Chem. 1957;226:497-509.

36. Melo AM, Benatti RO, Ignacio-Souza LM, et al. Hypothalamic endoplasmic reticulum stress and insulin resistance in offspring of mice dams fed highfat diet during pregnancy and lactation. Metabolism. 2014;63:682-92. doi:10. 1016/j.metabol.2014.02.002.

37. Payolla TB, Lemes SF, de Fante T, et al. High-fat diet during pregnancy and lactation impairs the cholinergic anti-inflammatory pathway in the liver and white adipose tissue of mouse offspring. Mol Cell Endocrinol. 2016;422:192202. doi:10.1016/j.mce.2015.12.004.

38. Reginato A, de Fante T, Portovedo M, et al. Autophagy proteins are modulated in the liver and hypothalamus of the offspring of mice with diet-induced obesity. J Nutr Biochem. 2016;34:30-41. doi:10.1016/j.jnutbio.2016.04.002.

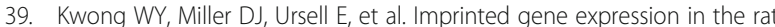
embryo-fetal axis is altered in response to periconceptional maternal low protein diet. Reproduction. 2006;132:265-77. doi:10.1530/rep.1.01038.

40. Brameld JM, Mostyn A, Dandrea J, et al. Maternal nutrition alters the expression of insulin-like growth factors in fetal sheep liver and skeletal muscle. J Endocrinol. 2000;167:429-37.

41. Zhang J, Zhang F, Didelot $X$, et al. Maternal high fat diet during pregnancy and lactation alters hepatic expression of insulin like growth factor-2 and key microRNAs in the adult offspring. BMC Genomics. 2009;10:478. doi:10. 1186/1471-2164-10-478.

42. Priego T, Sánchez J, García AP, et al. Maternal dietary fat affects milk fatty acid profile and impacts on weight gain and thermogenic capacity of suckling rats. Lipids. 2013;48:481-95. doi:10.1007/s11745-013-3764-8.

43. Sears B, Perry M. The role of fatty acids in insulin resistance. Lipids Health Dis. 2015;14:121. doi:10.1186/s12944-015-0123-1.
44. Zarfeshani A, Ngo S, Sheppard AM. MicroRNA Expression Relating to DietaryInduced Liver Steatosis and NASH. J Clin Med. 2015:4:1938-50. doi:10.3390/ jcm4111938.

45. Yang WM, Jeong HJ, Park SY, Lee W. Saturated fatty acid-induced miR-195 impairs insulin signaling and glycogen metabolism in HepG2 cells. FEBS Lett. 2014;588:3939-46. doi:10.1016/j.febslet.2014.09.006.

46. Nie Y-Q, Cao J, Zhou Y-J, et al. The effect of miRNA-122 in regulating fat deposition in a cell line model. J Cell Biochem. 2014;115:839-46. doi:10. 1002/jcb.24725.

47. Sun B, Purcell RH, Terrillion CE, et al. Maternal High-Fat Diet During Gestation or Suckling Differentially Affects Offspring Leptin Sensitivity and Obesity. Diabetes. 2012;61:2833-41. doi:10.2337/db11-0957.

48. Cerf ME, Williams $\mathrm{K}, \mathrm{Nkomo} \mathrm{Xl}$, et al. Islet cell response in the neonatal rat after exposure to a high-fat diet during pregnancy. Am J Physiol Regul Integr Comp Physiol. 2005;288:R1122-8. doi:10.1152/ajpregu.00335.2004

49. Okun N, Verma A, Mitchell BF, Flowerdew G. Relative importance of maternal constitutional factors and glucose intolerance of pregnancy in the development of newborn macrosomia. J Matern Fetal Med. 1997;6:285-90. doi:10.3109/14767059709162004.

50. Baeten JM, Bukusi EA, Lambe M. Pregnancy complications and outcomes among overweight and obese nulliparous women. Am J Public Health. 2001:91:436-40. doi:10.2105/AJPH.91.3.436.

51. Skarzinski G, Khamaisi M, Bursztyn M, et al. Intrauterine growth restriction and shallower implantation site in rats with maternal hyperinsulinemia are associated with altered NOS expression. Placenta. 2009;30:898-906. doi:10. 1016/j.placenta.2009.07.014.

52. Khamaisi M, Skarzinski G, Mekler J, et al. Hyperinsulinemia increases placenta endothelin-converting enzyme-1 expression in trophoblasts. Am J Hypertens. 2012;25:109-14. doi:10.1038/ajh.2011.164

53. Kahraman S, Dirice E, De Jesus DF, et al. Maternal insulin resistance and transient hyperglycemia impact the metabolic and endocrine phenotypes of offspring AJP Endocrinol Metab. 2014;307:E906-18. doi:10.1152/ajpendo.00210.2014.

54. Brumbaugh DE, Friedman JE. Developmental origins of nonalcoholic fatty liver disease. Pediatr Res. 2014;75:140-7. doi:10.1038/pr.2013.193.

55. McCurdy CE, Bishop JM, Williams SM, et al. Maternal high-fat diet triggers lipotoxicity in the fetal livers of nonhuman primates. J Clin Invest. 2009;119: 323-35. doi:10.1172/JCl32661

56. Hsu SH, Wang B, Kota J, et al. Essential metabolic, anti-inflammatory, and anti-tumorigenic functions of miR-122 in liver. J Clin Invest. 2012;122:287183. doi:10.1172/JCl63539.

57. Xu W-P, Yi M, Li Q-Q, et al. Perturbation of MicroRNA-370/Lin-28 homolog A nuclear factor kappa B regulatory circuit contributes to the development of hepatocellular carcinoma. Hepatology. 2013;58:1977-91. doi:10.1002/hep.26541.

58. Silber GH, Hachey DL, Schanler RJ, Garza C. Manipulation of maternal diet to alter fatty acid composition of human milk intended for premature infants. Am J Clin Nutr. 1988:47:810-4.

59. Novak EM, Innis SM. Impact of maternal dietary n-3 and n-6 fatty acids on milk medium-chain fatty acids and the implications for neonatal liver metabolism. AJP Endocrinol Metab. 2011;301:E807-17. doi:10.1152/ajpendo.00225.2011.

60. Thorn SR, Baquero KC, Newsom SA, et al. Early life exposure to maternal insulin resistance has persistent effects on hepatic NAFLD in juvenile nonhuman primates. Diabetes. 2014;63:2702-13. doi:10.2337/db14-0276.

61. Stewart MS, Heerwagen MJ, Friedman JE. Developmental Programming of Pediatric Non-Alcoholic Fatty Liver Disease: Redefining the "First-Hit.". Clin Obs Gynecol. 2013;56:577-90. doi:10.1097/GRF.0b013e3182a09760.

62. Day CP, James OF. Steatohepatitis: a tale of two "hits"? Gastroenterology. 1998;114:842-5.

63. Tilg H, Moschen AR. Evolution of inflammation in nonalcoholic fatty liver disease: The multiple parallel hits hypothesis. Hepatology. 2010;52:1836-46. doi:10.1002/hep.24001.

64. de Fante T, Simino LA, Reginato A, et al. Diet-Induced Maternal Obesity Alters Insulin Signalling in Male Mice Offspring Rechallenged with a High-Fat Diet in Adulthood. PLoS One. 2016;11:e0160184. doi:10.1371/journal.pone.0160184.

65. Glavas MM, Kirigiti MA, Xiao XQ, et al. Early overnutrition results in earlyonset arcuate leptin resistance and increased sensitivity to high-fat diet. Endocrinology. 2010;151:1598-610. doi:10.1210/en.2009-1295. 